Boise State University

ScholarWorks

Materials Science and Engineering Faculty

Publications and Presentations

Department of Materials Science and Engineering

8-1-2007

\title{
Determination of Three-Dimensional Grain Boundary Connectivity from Two-Dimensional Microstructures
}

Megan Frary

Boise State University

\section{(1) $(1 \otimes \Theta$}

This is an author-produced, peer-reviewed version of this article. (C) 2009, Elsevier. Licensed under the Creative Commons AttributionNonCommercial-NoDerivatives 4.0 International License (https://creativecommons.org/licenses/by-nc-nd/4.0/). The final, definitive version of this document can be found online at Scripta Materialia, doi: 10.1016/j.scriptamat.2007.04.008 


\title{
Determination of Three-Dimensional Grain Boundary Connectivity from Two-Dimensional Microstructures
}

\author{
Megan Frary \\ Materials Science and Engineering, Boise State University, Boise, ID 83725-2075
}

\begin{abstract}
The connectivity of so-called "special" and "general" grain boundaries at a quadruple node is known to be nonrandom as a result of crystallographic constraints. Although a quadruple node is a three-dimensional feature, there exist two-dimensional features which are topologically identical. Therefore, the distribution of these twodimensional features may be used to determine the three-dimensional connectivity. Computer simulations of a three-dimensional microstructure which is virtually serial sectioned are used to validate the proposed approach.
\end{abstract}

Keywords: Grain boundaries; microstructure.

\section{Introduction}

Grain boundaries are known to play an important role for many properties [1]. Although the rigorous definition of grain boundary structure has five macroscopic degrees of freedom [1], it is common to apply a binary classification on the basis of the boundary structure [2,3]. The "special" boundaries are those that are resistant to damage (e.g., cracking), while "general" boundaries are susceptible to damage $[4,5]$. It has recently been proposed that the properties of a material depend not only on the fraction of special boundaries, but also on the connectivity of these damage-resistant boundaries [6-8]. One quantitative approach used to study grain boundary connectivity is the triple junction distribution (TJD) [9-11], which gives $\mathrm{J}_{\mathrm{i}}$, the fraction of junctions coordinated by $i$ (= $0,1,2$ or 3) special boundaries. If the special and general boundaries have a random spatial distribution, the TJD is found using a probabilistic argument as:

$$
J_{i}=\left(\begin{array}{c}
3 \\
i
\end{array}\right) p^{i}(1-p)^{3-i}
$$

where $p$ is the global fraction of special boundaries, and the combinations $\left(\begin{array}{l}3 \\ \mathrm{i}\end{array}\right)$ are equal to $1,3,3$, and 1 for $i=0,1,2$ and 3, respectively. The TJD can be determined easily from a two-dimensional (2-D) section of a microstructure; however, when compared to the prediction of Eq. (1), the TJDs in real materials are found to be highly nonrandom $[9,10,12]$. While $J_{1}$ junctions are more abundant than predicted, $\mathrm{J}_{2}$ junctions are scarcer. The deviation from Eq. (1) is due to the requirement for crystallographic consistency around any closed path in the microstructure $[9,10]$. If a circuit is drawn which crosses a number of grain boundaries and which ends in the same grain where it began, the changes in misorientation must sum to zero (i.e., misorientation is conserved). The smallest such path encircles a triple junction and is referred to as a first-order constraint [13].

The connectivity of grain boundaries in threedimensional (3-D) microstructures is of more practical interest as materials are inherently three-dimensional. In 3$\mathrm{D}$, the local special boundary coordination can be studied at quadruple nodes, the points where four grains, six grain boundaries and four triple junctions meet. Figure 1a shows an illustration of a quadruple node where the grains are modeled as tetrakaidecahedra and labeled $G_{0}, G_{1}, G_{2}$ and $\mathrm{G}_{3}$. Similar to triple junctions, quadruple nodes can be classified on the basis of their special boundary coordination and a quadruple node distribution (QND) defined as the population of quadruple nodes of a given type [14]. The quadruple node populations are labeled $\mathrm{Q}_{\mathrm{i}+\mathrm{j}}$, where $i(=0$ to 6$)$ is the number of special boundaries in the quadruple node and $j(=0$ to 4$)$ the number of triple junctions with two or more special boundaries. The QND can also be predicted for the unphysical case where boundaries are randomly assigned as either special or general as:

$$
\mathrm{Q}_{\mathrm{i}+\mathrm{j}}=\Phi_{\mathrm{i}+\mathrm{j}} \cdot \mathrm{p}^{\mathrm{i}}(1-\mathrm{p})^{6-\mathrm{i}}
$$

where $\Phi_{i+j}$ is the number of possible configurations for each node type and is equal to $1,6,3,12,4,12,4,12,3,6$ and 1 for $i+j=0,1,2,3,4,5,6,7,8,9$ and 10 , respectively.

Computer simulations have shown that not only is the QND nonrandom, but it cannot be predicted solely on the basis of the first-order (i.e., triple junction) constraints alone [14]. As quadruple nodes are comprised of four triple junctions, four first-order circuits exist. In addition, there are second-order constraints present, represented by circuits that begin and end in the same grain and that cross four of the six grain boundaries, which impose a higher degree of constraint on the special boundary coordination. Accordingly, the local correlations in 3-D microstructures cannot be predicted from the easily-acquired TJD alone. 

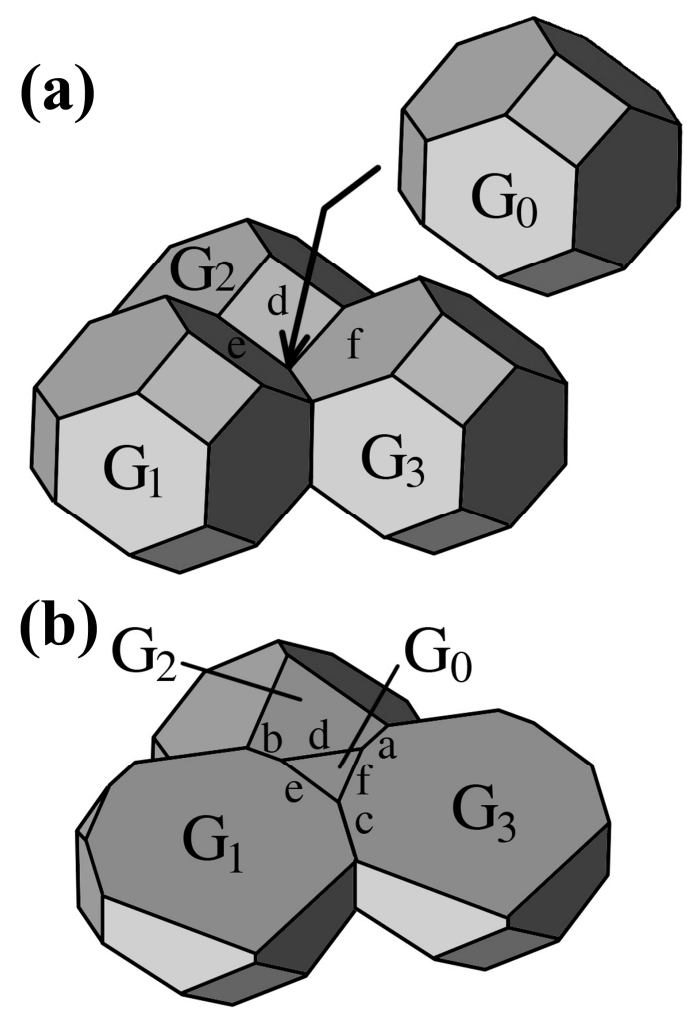

Figure 1: (a) The four grains, labeled $\mathrm{G}_{\mathrm{i}}$, which meet at a quadruple node. (b) If the grains are sectioned just above the quadruple node, grain $G_{0}$ appears as a three sided grain embedded in the other three grains. The six boundaries that comprise the QN, labeled $a$ through $f$, are also present around the three-sided grain.

As the connectivity and local correlations in grain boundary networks are becoming the focus of more work, it is important to be able to determine the QND in a straightforward manner. To the best of our knowledge, no experimental studies of the QND exist, although methods such as serial sectioning could be used. Unfortunately, serial sectioning and related techniques are time-consuming and do not permit rapid evaluation of the 3-D connectivity of a microstructure.

Although a quadruple node cannot be observed on a single 2-D microstructural section, the connectivity of boundaries at a quadruple node can be mapped onto a plane [14], such that particular features in 2-D sections may be topologically identical to a quadruple node, allowing for easy determination of the QND. We propose here a method by which the QND can be obtained by studying the statistical distribution of such 2-D features and compare the results to the QND determined for a 3-D microstructure.

\section{Geometrical approach}

To determine what 2-D feature may inform the 3-D connectivity, it is useful to consider how the features of a quadruple node might appear in a 2-D section. For example, if the grains in Figure 1a are sectioned above the quadruple node, the 2-D section shows all four grains and six grain boundaries in the quadruple node (Fig. 1b). Here, grain $\mathrm{G}_{0}$ appears as a three-sided grain whose neighboring grains are $G_{1}, G_{2}$ and $G_{3}$. Not only does the 2-D section in Figure $1 \mathrm{~b}$ reveal all of the features of the quadruple node, but the connectivity among grain boundaries is preserved. In other words, the same first- and second-order circuits that could be drawn through three or four grains in the quadruple node can also be drawn on the 2-D section as illustrated in Figure 2. In both cases, there are four unique first-order circuits that each passes through three grains and three grain boundaries. Labeling the circuits by the grain boundaries which they cross, the first-order circuits are a-bc, a-d-f, b-d-e, and c-e-f (Fig. 2a); the three second-order circuits are: c-e-d-a, b-d-f-c, and a-f-e-b (Fig. 2b). As the connectivity of the grain boundaries at a three-sided grain is identical to that of a quadruple node, the coordination of special boundaries around three-sided grains can be quantified by $\mathrm{T}_{\mathrm{i}+\mathrm{j}}$, the distribution of three-sided grain configurations which account for the special boundary populations, in direct analogy to the QND. These two distributions, $T_{i+j}$ and $Q_{i+j}$, should then be identical as they measure the special boundary coordination of topologically indistinguishable features. It should be emphasized that we do not suggest that all three-sided grains are associated with quadruple nodes, only that identical higher-order circuits which exist around a quadruple node are also present around three-sided grains, thus yielding the same
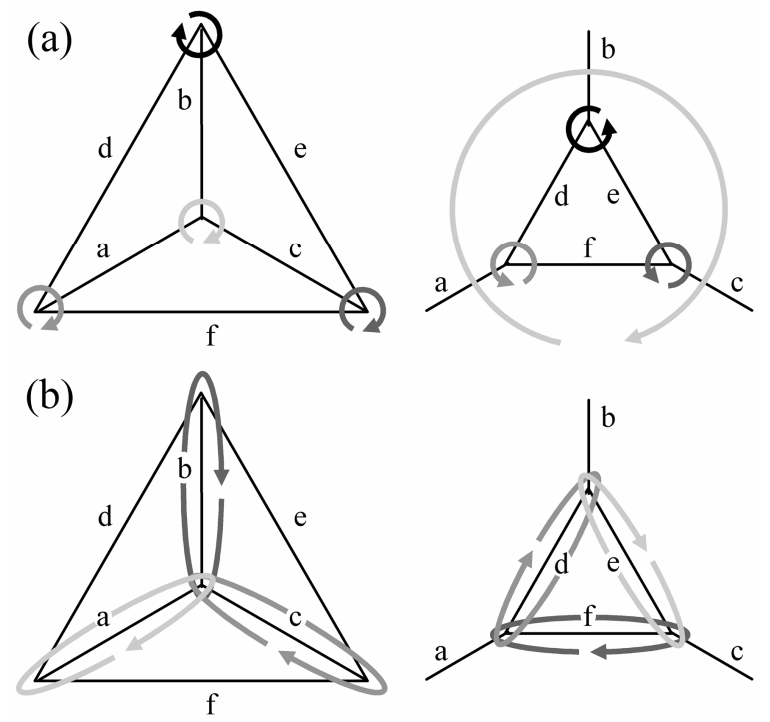

Figure 2: Schematic illustration of (a) first-order and (b) second-order constraints present at a quadruple node (left column) and three-sided grain (right column). The grain boundaries are labeled $a$ through $f$. The circuits which comprise each constraint are indicated by the arrows; firstorder circuits pass through three grains and three grain boundaries, while second-order circuits pass through four grains and four grain boundaries. 
information about the connectivity and allowing the QND, a property of a 3-D microstructure, to be obtained from a 2D section.

\section{Simulation procedure}

To validate the approach of classifying three-sided grains to obtain information on the 3-D connectivity of the microstructure, we simulated 3-D, equiaxed microstructures from which the quadruple node and threesided grain distributions could be obtained over all values of the special boundary fraction. The simulated microstructures used a 3-D array of volume elements in a close-packed, face-centered cubic configuration with $\mathrm{L}=$ 200 volume elements per side ( 8 million in total). Periodic boundary conditions were applied so that there were no edge effects. An equiaxed grain structure was created by randomly selecting $\Omega=1600$ volume elements as the grain seeds with arbitrary orientations labeled 0 to $\Omega-1$. Each of the $\mathrm{L}^{3}$ volume elements was then assigned the same orientation as the seed to which it was closest. Figure $3 \mathrm{a}$ shows a small structure simulated with this routine.

A total of 100 different simulated microstructures were constructed; for each, the distribution of grain boundary types was changed by reassigning the crystallographic orientation of each grain to affect a different special boundary fraction. Two distinct crystallographic textures were used, one which varied from an ideally random texture to a single crystal texture and another which varied from an ideal fiber texture to a single crystal texture; the methods for assigning grain orientations are described elsewhere [9]. In the following discussion, these microstructures will be referred to as general textured and fiber textured, respectively.

Once the grain orientations were assigned, grain boundary misorientations were calculated and boundaries with misorientations less than a threshold value were classified as special; all other boundaries were labeled general. The quadruple node distribution was obtained by scanning the microstructure for points where four grains and six grain boundaries met. The quadruple node character was determined from the number of special boundaries that met at the node and the number of triple junctions with at least two special boundaries. In order to determine the three-sided grain distribution from a twodimensional section (Fig. 3b), virtual serial sectioning was performed on each microstructure and data sets were extracted from the 3-D structure consisting of a single plane of volume elements. Two hundred planes were extracted normal to each of the three principal directions. Three-sided grains were identified (e.g., the circled grain in Fig. 3b) and the character of the three-sided grain configuration determined from the number of special boundaries present and the number of triple junctions with at least two special boundaries.
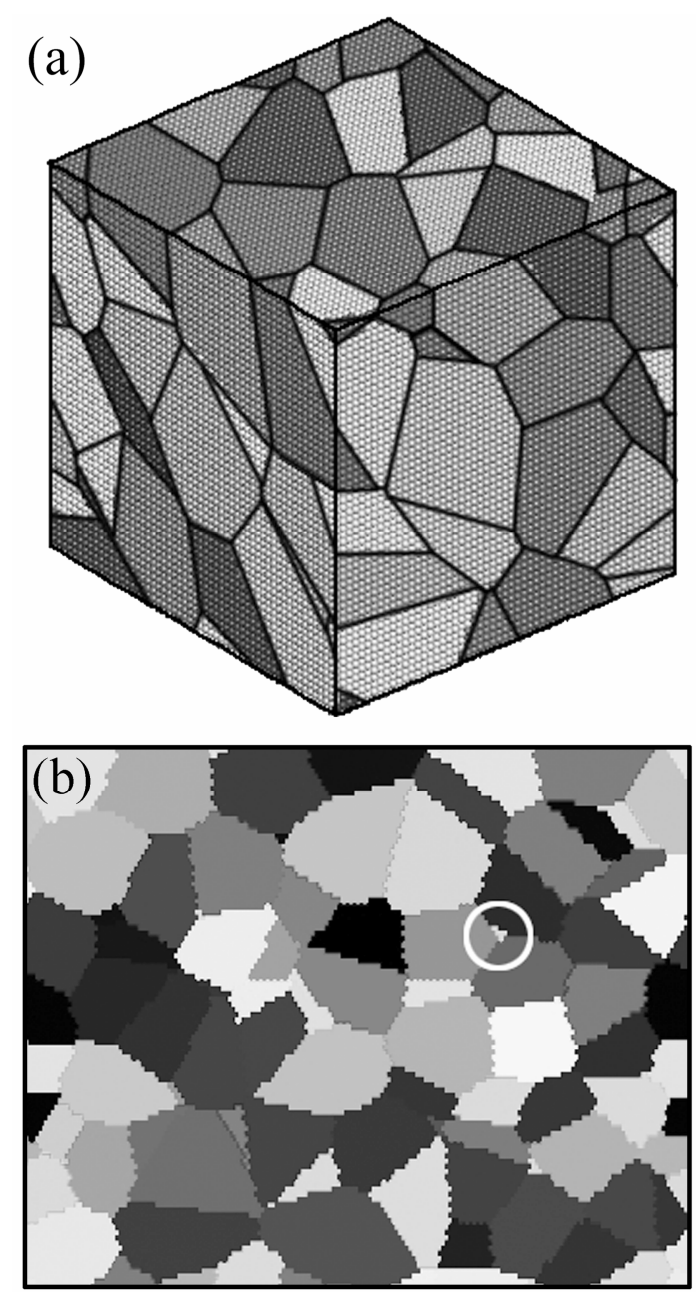

Figure 3: (a) Representative 3-D microstructural model with an equiaxed grain structure. (b) Virtual serial section through a similar microstructural model in which a threesided grain has been identified.

\section{Results and discussion}

During the simulations, the values $Q_{i+j}$ and $T_{i+j}$ were determined over all values of $p$ for each of the 100 simulated microstructures. For each microstructural realization, the values of $\mathrm{Q}_{\mathrm{i}+\mathrm{j}}$ and $\mathrm{T}_{\mathrm{i}+\mathrm{j}}$ can be plotted as a function of the special boundary fraction. Figure 4 shows one such plot for the population of nodes with $i+j=1$. For both fiber textured and general textured microstructures, $\mathrm{Q}_{1}$ (triangles) and $\mathrm{T}_{1}$ (circles) show good agreement. The dashed line in Figure 4 represents the prediction of Eq. (2) when boundaries are randomly assigned as special or general. However, it is clear that $\mathrm{Q}_{1}$ and $\mathrm{T}_{1}$ for the crystallographically-consistent microstructures do not match the prediction of Eq. (2). Figure 4 also shows the value of $Q_{1}$ from our previous simulations where the boundaries were modeled as the faces of polyhedra [14]. While the simulation methods differ, both $\mathrm{Q}_{1}$ and $\mathrm{T}_{1}$ match the previous simulations. 


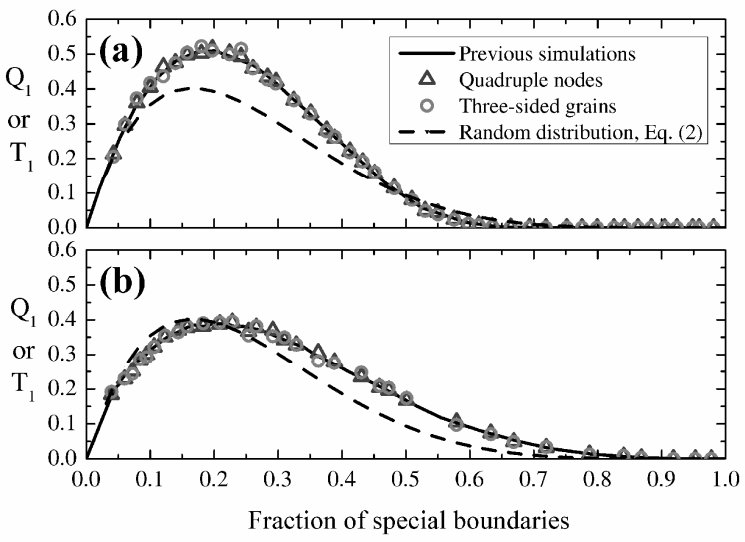

Figure 4: Quadruple node $(\triangle)$ and three-sided grain $(O)$ distributions as a function of special boundary fraction. The dashed line is for a randomly-assembled microstructure as given by Eq. (2). The solid black lines correspond to the quadruple node distribution found previously [14] and serves as a basis for comparison.

Although not included here, the distributions for the other node types $\mathrm{Q}_{i+j}$ and $\mathrm{T}_{\mathrm{i}+\mathrm{j}}$ for this and other microstructural realizations match equally well to one another and to prior simulations. The consistency among $\mathrm{Q}_{\mathrm{i}+\mathrm{j}}$ and $\mathrm{T}_{\mathrm{i}+\mathrm{j}}$ suggests that the quadruple node distribution can be found by analyzing the statistical distribution of three-sided grains in a two-dimensional microstructural section. In order to achieve the accuracy observed here, approximately 2500 three-sided grains must be analyzed. The present simulations and concurrent experimental work suggest that $\sim 9 \%$ of grains in a 2-D section appear three-sided; therefore, for a fine-grained specimen, an accurate QND could be found by analyzing a few square millimeters.

Although Figure 4 suggests that $T_{i+j}$ is equivalent to $Q_{i+j}$, differences between the two exist. In order to evaluate how good an approximation $\mathrm{T}_{\mathrm{i}+\mathrm{j}}$ is for $\mathrm{Q}_{\mathrm{i}+\mathrm{j}}$, the difference, $\Delta_{\mathrm{i}+\mathrm{j}}$, between the two for each value of $i+j$ was analyzed for each of the 100 microstructural realizations. The difference is defined as:

$$
\Delta_{\mathrm{i}+\mathrm{j}}=\left|\mathrm{Q}_{\mathrm{i}+\mathrm{j}}-\mathrm{T}_{\mathrm{i}+\mathrm{j}}\right|
$$

and was determined for every value of the special boundary fraction. After finding all values of $\Delta$ for all realizations (approximately 10,000 values), a cumulative distribution was plotted for $\Delta$. This process was repeated for each $i+j$ from 0 to 10 and for fiber textured and general textured microstructures. From the cumulative distributions, the magnitude of $\Delta$ below which $90 \%, 98 \%$ and $100 \%$ of differences fell could be determined. As Figure 5 indicates, $90 \%$ of the differences for a given $i+j$ were often substantially below 0.01 and, in most cases, $98 \%$ of the errors were below 0.015 . While the maximum values of $\Delta$ are $\sim 0.03$, the number of points with such differences was exceedingly small. Furthermore, it is useful to note that the differences observed here are smaller than the statistical errors would be in determining such distributions from real microstructures. Therefore, we find that $\mathrm{Q}_{i+j}$ may be reliably predicted from $T_{i+j}$.

Finally, it is important to mention that the differences calculated here are between the measured values of $Q_{i+j}$ and $\mathrm{T}_{\mathrm{i}+\mathrm{j}}$. In other words, the values are being compared to one another, rather than comparing the deviations of both to a known baseline (e.g., an analytical value for $\mathrm{Q}_{i+j}$ ). Although the latter may be more appropriate, an analytical expression is not available for fiber textured or general textured microstructures.

\section{Conclusions}

We have studied the connectivity of grain boundaries in three dimensions using two-dimensional microstructures. The main conclusions are:

1. Three-sided grains and quadruple nodes are topologically identical. In other words, the same firstand second-order crystallographic constraints exist at both three-sided grains and quadruple nodes.

2. The connectivity of special boundaries at a quadruple node, quantified through the QND, can be obtained by evaluating the distribution of three-sided grain types in a 2-D microstructure.

3. Although small differences occurred between the

\section{$90 \%, \square 98 \%, \quad \square 100 \%$ of errors}

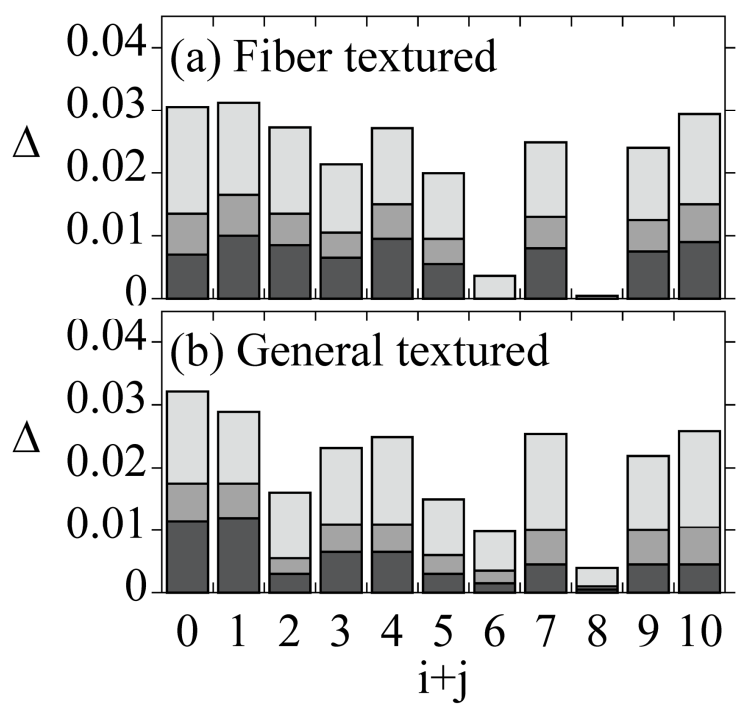

Figure 5: Magnitude of differences, $\Delta$, between the quadruple node distribution $\left(Q_{i+j}\right)$ from the 3-D microstructure and the three-sided grain distribution $\left(T_{i+j}\right)$ from 2-D sections through the microstructure. The grains were assigned with (a) fiber texture or (b) general texture. In general, $90 \%$ of differences are smaller than 0.01 . 
distributions $\mathrm{Q}_{\mathrm{i}+\mathrm{j}}$ and $\mathrm{T}_{\mathrm{i}+\mathrm{j}}$, the differences were almost always less than 0.01, an accuracy which exceeds what could be achieved experimentally.

4. The analysis technique described here has been validated with computer simulations of 3-D microstructures and virtual serial sectioning, and could be readily applied to experimental data sets. This work is underway to predict quadruple node distributions for real materials.

\section{Acknowledgements}

The author would like to thank Professor C.A. Schuh at MIT for useful discussions and Professor A. Jain at Boise State University for his help converting and optimizing the simulation algorithms.

\section{References}

[1] A. P. Sutton, R. W. Balluffi. Interfaces in Crystalline Materials,Oxford, 1995.
[2] G. Palumbo, K. T. Aust, E. M. Lehockey, U. Erb, P. Lin, Scripta Mater. 38 (1998) 1685.

[3] V. Randle, Acta Mater. 52 (2004) 4067.

[4] M. Shimada, H. Kokawa, Z. J. Wang, Y. S. Sato, I. Karibe, Acta Mater. 50 (2002) 2331.

[5] T. Watanabe, Mater. Sci. Eng. A176 (1994) 39.

[6] C. A. Schuh, M. Kumar, W. E. King, Acta Mater. 51 (2003) 687.

[7] G. Wang, L. Zuo, C. Esling, Phil. Mag. A82 (2002) 2499.

[8] A. J. Henrie, B. L. Adams, R. J. Larsen, Mater. Sci. Forum 408-412 (2002) 419.

[9] M. Frary, C. A. Schuh, Phys. Rev. B 69 (2004) 134115.

[10] R. W. Minich, C. A. Schuh, M. Kumar, Phys. Rev. B 66 (2002) 052101.

[11]P. Fortier, W. A. Miller, K. T. Aust, Acta Mater. 45 (1997) 3459.

[12] C. A. Schuh, M. Kumar, J. Mater. Sci. 40 (2005) 847.

[13]C. A. Schuh, M. Frary, Scripta Mater. 54 (2006) 1023.

[14] M. Frary, C. A. Schuh, Phil. Mag. 85 (2005) 1123. 